tin und Pemetrexed konnte eine objektive Ansprechrate von $55 \%$ im Vergleich zu $29 \%$ unter der Chemotherapie allein beobachtet werden", so Langer $(\mathrm{p}=0,0016)$. Die Ansprechrate habe sich im Vergleich zum derzeitigen Behandlungsstandard damit nahezu verdoppelt. Zudem reduzierte sich im Pembrolizumab-Arm das Progressions- oder Mortalitätsrisiko gegenüber der alleinigen Chemotherapie signifikant (HR 0,53, 95\%-KI 0,31-0,91; p = 0,012). „Die vergleichsweise gut verträgliche Kombinationstherapie mit Pembrolizumab könnte somit in Zukunft eine wirksame Behandlungsoption für Patienten mit Chemotherapie-naivem, fortgeschrittenem nichtplattenepithelialem NSCLC werden", schlussfolgerte Langer.

Silke Wedekind

\section{PD1-Blockade auch bei Kopf-Hals- Tumoren effektiv}

Patienten mit rezidivierenden oder metastasierenden platinresistenten Plattenepithelkarzinomen im Kopf-Hals-Bereich (SCCHN, „squamous cell carcinoma of the head and neck") haben eine Lebenserwartung von weniger als sechs Monaten. „Die Lebensqualität der Betroffenen während dieser Zeit zu erhalten, in der sie oft stark an den Symptomen ihrer Erkrankung sowie den Therapiefolgen leiden, ist ein zentrales Behandlungsziel“, erläuterte Kevin Harrington, London, Großbritannien.

In der Phase-III-Studie CheckMate 141 war das mediane OS von Patienten mit fortgeschrittenem SCCHN unter einer Therapie mit dem PD-1-Blocker Nivolumab im Vergleich zu einer Standard-Chemotherapie nach Wahl des Behandlers bei besserer Verträglichkeit signifikant länger (7,5 vs. 5,1 Monate; HR 0,70, $95 \%$-KI 0,51-0,96; $\mathrm{p}=0,01$ ) [Ferris RL et al. N Engl J Med. 2016 Oct 8. (Epub ahead of print)]. Nun wurden erstmals ergänzend die explorativ untersuchten "patient reported outcomes“ (PRO) aus dieser Studie vorgestellt [Harrington $\mathrm{K}$ et al. ESMO. 2016; Abstr LBA4]. Die PRO wurden mithilfe von drei Lebensqualitätsfragebögen zu Studienbeginn, in Woche 9 und dann im Abstand von sechs Wochen während der Behandlung erfasst (EORTC QLQ-C30, EORTC QLQ-H\&N35, EQ-5D-3L). Ana-

\title{
Adjuvante Immuntherapie mit Ipilimumab: effektiv, aber nebenwirkungsreich
}

Die adjuvante Therapie mit Ipilimumab verbessert bei Melanompatienten im Stadium III nicht nur das rezidivfreie Überleben (RFS) signifikant, sondern auch das Gesamtüberleben (OS). Das zeigt die aktualisierte Analyse der PhaseIII-Studie EORTC 18071, die Alexander Eggermont, Paris, Frankreich, auf der ESMO-Jahrestagung 2016 vorstellte [Eggermont A et al. ESMO. 2016;Abstr LBA2_PR]. In dieser Situation bestehe weiterhin großer therapeutischer Bedarf, da die adjuvante Therapie mit Interferon $\mathrm{a} 2 \mathrm{~b}$ nur zu einer moderaten Verbesserung des Überlebens führe, so Eggermont. Deshalb wurde Ipilimumab in der placebokontrollierten Studie als adjuvante Therapie bei 951 Hochrisikopatienten im Stadium III nach kompletter Tumorresektion geprüft. Der Antikörper wurde zunächst als Induktionstherapie, danach maximal drei Jahre lang als Erhaltungstherapie verabreicht. Die Studie hatte ihren primären Endpunkt bereits bei der ersten Auswertung erreicht [Eggermont $A$ et al. Lancet Oncol. 2015;16(5):522-30]: Ipilimumab führte gegenüber Placebo zu einem signifikant längeren RFS (26,1 vs. 17,1 Monate; $p=0,0013$ ).

Nach jetzt median 5,3-jährigem Follow-up sind auch die Daten zum Gesamtüberleben ausgereift. Das Update bestätigt den RFS-Benefit der adjuvanten Ipilimumab-Therapie gegenüber Placebo (27,6 vs. 17,1 Monate; Hazard Ratio [HR] 0,76; $p=0,0008$ ). Auch beim Gesamtüberleben war Ipilimumab signifikant überlegen: Die 5-Jahresrate betrug $54 \%$ im Placeboarm und $65 \%$ bei aktiver Therapie (HR 0,72; $p=0,001)$. In beiden Armen ist der Überlebensmedian noch nicht erreicht. Mit Ausnahme von Patienten im Stadium IIIA profitierten alle Subgruppen von der adjuvanten Ipilimumab-Therapie. Auch beim metastasenfreien Überleben schnitten Patienten des Verumarms signifikant besser ab als Patienten im Placebo-Arm (HR 0,76; $p=0,002$ ).

"In der adjuvanten Situation ist die Sicherheit der Therapie vorrangig", betonte Eggermont. 45,4\% der Patienten erlebten schwere Nebenwirkungen vom Grad 3/4 unter der aktiven Therapie; $33 \%$ brachen diese wegen Nebenwirkungen ab. Die Rate schwerer immunbedingter Nebenwirkungen betrug 41,6\%. Drei Patienten starben an einer immunbedingten Kolitis, einer an einem Guillain-Barré-Syndrom. Als schwerwiegend bezeichnete Eggermont auch Hypophysitiden, die eine sehr lange bis lebenslange Hormonersatztherapie erfordern. Er wies zudem darauf hin, dass nur $13,4 \%$ der Patienten im Verumarm die Ipilimumab-Erhaltungstherapie wie vorgesehen über die gesamten drei Jahre erhielten (Placebo: 30,2\%). Hauptgrund für einen Therapieabbruch waren Nebenwirkungen bei fast jedem zweiten Patienten.

Abschließend wertete Eggermont die adjuvante Ipilimumab-Gabe als wichtige neue Option für HochrisikoMelanompatienten im Stadium III. Auch Diskutant Olivier Michielin, Lausanne, Schweiz, bezeichnete die EORTC-Studie als wichtigen Meilenstein in der Melanombehandlung. Er wies jedoch auf die nicht unbeträchtliche Toxizität hin. Seiner Meinung nach sollte die Therapie daher erfahrenen Zentren vorbehalten bleiben.

Katharina Arnheim lysiert werden konnten die Daten von 129 Patienten, die die Fragebögen zu Studienbeginn und im Laufe der Nachbeobachtung ausgefüllt hatten. „Während bei Patienten des Nivolumab-Arms sowohl die Symptomlast als auch die Funktionalität nach 9 und 15 Wochen stabil geblieben war oder sich sogar verbessert hatte, erfuhren Patienten des Standardchemotherapie-Arms eine Verschlechterung in allen Domänen der untersuchten Lebensqualitäts-Scores“, sagte Harrington. „Damit ist Nivolumab der erste Checkpointinhibitor, für den bei Patienten mit fortgeschrittenem SCCHN ein signifikanter Überlebensvorteil bei besserer Verträglichkeit und besserem Erhalt der Lebensqualität im Vergleich zur Standardtherapie nachgewiesen werden konnte“, schloss Harrington.

Silke Wedekind

\section{Neue Option bei HR-positivem Brustkrebs}

Beim metastasierten Hormonrezeptor (HR)-positiven Brustkrebs ist die Erstlinientherapie mit einem Aromatasehemmer derzeit Mittel der Wahl. Erreicht 